\title{
Abastecimiento de agua potable por camiones aljibe durante la megasequía. Un análisis hidrosocial de la provincia de Petorca, Chile
}

Maria-Christina Fragkou. Universidad de Chile, Santiago, Chile.

Tamara Monsalve-Tapia. Universidad de Chile, Santiago, Chile.

Vicente Pereira-Roa. Universidad de Chile, Santiago, Chile.

Maximiliano Bolados-Arratia. Universidad de Chile, Santiago, Chile.

RESUMEN | A pesar de su amplia extensión espacial y temporal, la distribución de agua mediante camiones aljibe (cisterna) no ha sido estudiada desde la Geografía. En este artículo hacemos una primera aproximación a este sistema transitorio y poco convencional de distribución hídrica, analizando el volumen, costo, trayectoria y los dueños de estos flujos de agua que se transportan a lo largo de la provincia de Petorca, a partir de 1.753 órdenes de compra de agua (2012-2018). Al mismo tiempo, contribuimos conceptualmente a la comprensión de los sistemas de abastecimiento de agua potable que no caben en la categoría de infraestructura u obra hidráulica, proponiendo el concepto de "red hidrosocial", que permite incluir las múltiples dimensiones y escalas de los sistemas de abastecimiento de agua de emergencia. Nuestros resultados demuestran la creación de un mercado que se basa en los excedentes de agua que pueden tener ciertos actores, incluso en zonas de extrema sequía.

PALABRAS ClAVE | redes, gestión ambiental, asentamientos humanos.

ABSTRACT | Despite of its wide spatial and temporal extension, the distribution of water by means of water trucks has not been studied from a geographical standpoint. In this article we make a first approximation to this ephemeral and unconventional water distribution system, analyzing the volume, cost, trajectory, and owners of these water flows that are transported throughout the Province of Petorca, based on 1,753 water purchase orders (2012-2018). At the same time, we contribute to the understanding of potable water supply systems that do not fit into the category of infrastructure or hydraulic works. We propose the concept of "hydro-social network", which allows for the inclusion of the multiple dimensions and scales of emergency water supply systems. Our results demonstrate the creation of a market based on the surpluses that certain actors may have, even in areas of extreme drought.

KEYWORDS | networks, environmental management, human settlements.

Recibido el 29 de diciembre de 2020, aprobado el 15 de marzo de 2021.

E-mails: M. Fragkou, mariac.fragkou@uchilefau.cl|T. Monsalve-Tapia, tamara.monsalve@ug.uchile.cl|V. Pereira-Roa, vpereiraroa@umass. edu | M. Bolados-Arratia, mbolados@renare.uchile.cl 


\section{Introducción}

En medio de una establecida crisis hídrica y una creciente crisis climática, tanto la clase política como la opinión pública, la prensa y la literatura académica han resaltado la dimensión social de la escasez hídrica, comprendida como un problema político, algo que la Ecología Política ha manifestado desde hace años y constantemente (Budds, 2009; Prieto, 2015). En Chile, una de las causas políticas de la escasez hídrica se detecta en el programa neoliberal de la dictadura, que necesitó la circulación del agua fuera de la influencia pública y estatal (Budds, 2020), saliendo así de la tragedia de los comunes, para entrar en la de la propiedad privada sobre los Derechos de Aprovechamiento de Aguas (DAA). Así, el agua, a través de estos DAA, terminó siendo vendida y comprada en un mercado desconectado de su territorio, con mínimas consideraciones ambientales y ninguna social, en un marco legal definido por la Constitución aprobada en 1980 y el Código de Aguas del año siguiente. Cabe destacar que, si bien este Código ha sido reformado posteriormente, estas reformas son consideradas modestas, con escasas modificaciones de los principios fundamentales de DAA de propiedad privada bajo los poderes del mercado y un Estado débil (Bauer, 2015). Este marco legislativo e institucional vigente ha resultado en una distribución cínicamente desigual, donde los derechos de agua son acumulados por parte de grandes empresas nacionales y multinacionales de minería y agroexportación y por el sector financiero, mientras las comunidades rurales se han quedado prácticamente sin agua (Urquiza \& Billi, 2020).

La escasez hídrica construida social o políticamente que viven los territorios rurales (Oppliger et al., 2019) implica la fragilidad de estas zonas frente a eventos climáticos extremos, como la prolongada megasequía que empezó el 2010 (Muñoz et al., 2020), y que hasta hoy condiciona la vida y salud de comunidades y ecosistemas. En este marco, el grave y prolongado problema de abastecimiento de agua potable en comunidades rurales y comités de Agua Potable Rural (APR) llevó al cuestionamiento de la política hídrica nacional, de los modos de producción agrícola exportador y de su impacto local (Panez-Pinto et al., 2017). Entre las soluciones estatales de emergencia, la distribución de agua potable para consumo humano mediante camiones aljibe ha sido una solución inmediata y permanente para sustituir el abastecimiento tradicional por cañerías, pozos o vertientes en comunidades principalmente rurales; y en una problemática diferente en los campamentos y las urbanizaciones a lo largo del país (Nazer et al., 2018). El año 2019, más de 383.000 hogares rurales vivían sin agua potable, mientras el 15,4\% de las comunidades rurales recibió agua de camiones aljibe (Fundación Amulén et al., 2019). Este sistema de distribución de agua para consumo humano es, sin embargo, un modo de abastecimiento hídrico precario, discontinuo y costoso, y amenaza el derecho humano al agua y la vida (Bravo \& Fragkou, 2019; Oppliger et al., 2019).

A pesar de su amplia extensión espacial y temporal, hasta ahora no se ha sistematizado, ni ha sido objeto de estudio desde la academia, la distribución de agua mediante camiones aljibe en las zonas impactadas por la megasequía, como tampoco se ha indagado en la cantidad, el costo, la procedencia y los dueńos de esta agua, a excepción de lo publicado en algunas notas periodísticas (Arellano, 2017; Equipo 
Ameonna, 2020). En este artículo cubrimos este vacío desde la Geografía, para analizar la red de distribución de agua mediante camiones aljibe en la provincia de Petorca, con base en los procesos administrativos que sostienen y organizan esta red, la trayectoria espacial de los flujos hídricos, su valor económico y los proveedores de esta agua, cuadro que se movilizó dentro de la misma provincia durante la megasequía (2010-2018).

Evaluamos nuestros resultados desde la literatura de la ecología política, que recalca y analiza la dimensión sociopolítica de los problemas ambientales y propone investigar los territorios como sistemas hidrosociales (Bolding, 2004; Hoogesteger \& Wester, 2015). Esta perspectiva logra captar la compleja y multidimensional realidad hídrica de los territorios, incorporando en un mismo análisis factores físicos, económicos, políticos y culturales, y develando tanto las múltiples causas de la escasez como los impactos de las soluciones destinadas a mitigarla. En esta misma línea, se definen como redes hidrosociales las redes socio-técnico-naturales que permiten la circulación espacio-temporal del agua y su domesticación, definiendo su acceso a la sociedad (Wester, 2008).

En este artículo proponemos la comprensión y análisis de los sistemas de distribución de agua por camiones aljibe como "redes hidrosociales", puesto que, por ser un sistema de distribución efímero, inestable y conformado por flujos aperiódicos de agua en movimiento motorizado, no se lo puede caracterizar como obra, infraestructura o capital hidráulico (Freire-González \& Puig-Ventosa, 2016). Además, como concepto analítico, nos facilita la comprensión e integración de los sistemas hidrológicos, humanos y tecnológicos que sostienen la circulación espacial y temporal de agua por camiones aljibe, mientras visibiliza el rol del mercado de DAA y las decisiones políticas acerca del agua potable, que definen su acceso.

Por lo tanto, la contribución de este trabajo es doble: por un lado, realizamos el primer análisis integral de la distribución de agua potable mediante camiones aljibe en Chile, teniendo como caso de estudio una de las zonas más afectadas por la megasequía, la provincia de Petorca. En segundo lugar, contribuimos conceptualmente a la descripción y análisis de los sistemas de abastecimiento de agua potable que no caben en la categoría de infraestructura u obra hidráulica; sostenemos que la complejidad de la red hidrosocial permite incluir las múltiples dimensiones y escalas de los sistemas de abastecimiento de agua de emergencia, temporales e itinerantes, como los camiones aljibe.

Para realizar nuestro análisis se recopilaron y tabularon 1.753 órdenes de compra de agua mediante camiones aljibe emitidas por la Gobernación Provincial de Petorca entre los años 2012 y 2018, obtenidas desde el portal Mercado Público y solicitudes de transparencia. Adicionalmente, verificamos el volumen y la localización de los DAA de los proveedores de agua, de acuerdo con las inscripciones identificadas en el Registro de Propiedad de Aguas (RPA) de los Conservadores de Bienes Raíces (CBR) con jurisdicción en la provincia en el periodo 2000-2017. Complementamos nuestros datos cuantitativos con cinco entrevistas a funcionarios públicos implicados en este proceso, realizadas durante el 2019. A partir de la información recopilada se generaron tres productos de análisis: un esquema burocrático del proceso administrativo a partir de las entrevistas; diagramas de distribución espacial de flujo de 
aguas, con la plataforma en línea sankeymatic.com; y mapas donde se visualizan los metros cúbicos extraídos y repartidos dentro la provincia según Sector Hidrogeológico de Aprovechamiento Común ${ }^{1}$ (shac) (Ministerio de Obras Públicas, 2014).

A continuación profundizamos en el concepto de red hidrosocial que guía nuestra investigación, presentando sus orígenes conceptuales y epistemológicos. El siguiente apartado describe la problemática de abastecimiento de agua potable en la provincia de Petorca durante la megasequía y los mecanismos de solicitud de agua mediante camiones aljibe como solución. Después presentamos los principales resultados sobre el precio, el volumen, la trayectoria y los dueńos de los flujos de agua movilizada por estos vehículos. El artículo sigue con la discusión de nuestros resultados, donde analizamos el sistema de distribución mediante camiones aljibe a partir de dos características de las redes hidrosociales: su alcance y su durabilidad. Concluimos con la revisión de nuestros hallazgos principales y sus repercusiones para la gestión de agua en Chile.

\section{Los territorios hidrosociales como marco analítico para evaluar los impactos de la gestión del agua}

En la tradición geográfica más amplia, el concepto de territorio ha evolucionado de ser percibido como un espacio geográfico asociado a, y definido por, criterios instrumentales, administrativos y tecnocráticos (Escobar, 2008; Hommes et al., 2016), a ser considerado un espacio más complejo, que es a la vez "judicial, político, económico, social, cultural, afectivo y físico"(Hommes \& Boelens, 2017, p. 72). Siguiendo esta misma línea, en este trabajo entendemos el territorio desde un enfoque político-ecológico, que lo define como "la organización y expresión socio-materialmente constituida y geográficamente delineada de y para el ejercicio del poder político" (Swyngedouw \& Boelens, 2018, p. 117). Sobre estas bases, Seemann (2016, p. 160) subraya la importancia del aspecto territorial en las cuestiones de gestión del agua y en las luchas por su control, "incluidos los derechos de propiedad, los diseños tecnológicos, las estructuras jurídicas y los discursos" acerca del agua. Por lo tanto, los territorios hidrosociales se conceptualizan como "la cuestionada materialización imaginaria y socioambiental de una red multiescalar con límites espaciales en la que los seres humanos, los flujos de agua, las relaciones ecológicas, la infraestructura hidráulica, los medios financieros, los arreglos legalesadministrativos y las instituciones y prácticas culturales se definen, alinean y movilizan interactivamente a través de sistemas de creencias epistemológicas, jerarquías políticas y discursos naturalizantes" (Boelens et al., 2016, p. 2).

Las contribuciones sobre los territorios hidrosociales se centran en arrojar luz sobre cómo la naturalización y el encuadre técnico de los problemas del agua esconden la naturaleza política de los modelos de gestión del agua y producen, por medios discursivos, legales, institucionales, tecnológicos e infraestructurales,

1 De acuerdo con el Decreto 203 del Ministerio de Obras Públicas de Chile, de 2014, un shaC es un "acuífero o parte de un acuífero cuyas características hidrológicas, espaciales y temporales permiten una delimitación para efectos de su evaluación hidrogeológica o gestión en forma independiente" (art. 54, letra g). 
territorios hidrosociales que mantienen las desigualdades sociales y la exclusión, y que dejan sin cuestionar el orden político dominante y los intereses económicos imperantes (Perramond, 2016). Son los proyectos hidráulicos y los arreglos institucionales-legales los que definen, impiden o facilitan la circulación del agua por los territorios y, por ende, el acceso al agua para las comunidades, modificando al mismo tiempo los espacios constituidos que comprenden los territorios hidrosociales (Hommes et al., 2016), con beneficios y cargas desiguales para los diferentes actores (Boelens et al., 2016). En este contexto, varios autores han propuesto la utilización del concepto de redes de agua (Bolding, 2004) o redes hidrosociales (Wester, 2008) para describir el tejido sociotécnico que permite la circulación del agua en los territorios (vistos como entidades hidrosociales). En el presente trabajo utilizamos este último concepto como unidad analítica sociotécnica para comprender la intencionalidad en la movilización del agua entre actores humanos y no humanos (Law, 1993), en escalas políticas, económicas, culturales y ecológicas más amplias (Boelens et al., 2016).

Utilizamos el marco analítico de estudio de las redes hidrosociales que proponen Hoogesteger y Wester (2015) para enmarcar estas redes en la economía política y las cadenas de producción que son dependientes al agua, y en los dominios y discursos que definen el acceso al agua y que permiten su acumulación por ciertos actores. En este sentido, el concepto de red hidrosocial va más allá de la propia infraestructura hidráulica, para incluir los actores que producen y consumen el agua, la misma agua, y el contexto político y discursivo que define el tipo de infraestructura y su alcance socioespacial, definido por intereses económicos, arreglos legales e institucionales y relaciones de poder a distintas escalas. Un análisis desde la red hidrosocial nos permite, entonces, visibilizar tanto las decisiones políticas tras la selección de la infraestructura, como también a los actores que se benefician o se excluyen de cada red y, por ende, del acceso al agua en el transcurso del tiempo. Cuando la red hidrosocial de un territorio no logra garantizar el acceso al agua a toda su población, las comunidades, actores y cuerpos excluidos experimentan escasez hídrica y deben surgir nuevas redes o medios que les abastezcan. Este es un problema común en las ciudades del Sur global, donde, ante la expansión urbana y el surgimiento constante de áreas informales periurbanas, las autoridades deben decidir entre ampliar las redes de distribución de agua existentes o recurrir a soluciones temporales y fuera de la red de distribución establecida y formal (Blomkvist et al., 2020). Estas redes secundarias suelen ser frágiles, efímeras y vulnerables, tal como ocurre con lo que Broto et al. (2012) describen como metabolismos hídricos informales que surgen en los sectores (peri)urbanos, en paralelo a la red formal de distribución de agua potable.

El concepto de red hidrosocial escapa de las categorías restringidas de la infraestructura u obra hidráulica, que suelen componerse de elementos estáticos en términos espaciales y temporales (Freire-González \& Puig-Ventosa, 2016), y nos permite describir sistemas de abastecimiento que son temporales y móviles, como la distribución de agua por camiones aljibe, las máquinas de venta de prepago (Blomkvist et al., 2020), o las bolsas de agua potable (Morinville, 2017). Los tres tipos de abastecimiento son formales y legítimos, e imprescindibles para complementar el abastecimiento de agua potable, pero la bibliografía internacional no ha alcanzado 
un consenso acerca de cómo caracterizar la distribución de agua mediante estos mecanismos; y es por ello que la red hidrosocial, en sus dimensiones espaciales, temporales y formas de flujo, permite entender un conjunto de elementos técnicos, humanos y naturales que definen las fuentes hídricas, los puntos de acceso y la cantidad y calidad de agua que circula a lo largo de un territorio, siendo adecuada su utilización para capturar la complejidad de este sistema informal y efímero de distribución de agua potable.

En términos analíticos, y para entender mejor el funcionamiento y el alcance de las redes hidrosociales, Bolding (2004) define dos características críticas que incluiremos en nuestro análisis: su alcance y su durabilidad. La primera hace referencia al alcance espacial, social, material e institucional de una red hidrosocial, y su extensión depende de la escala de análisis y asociaciones que se están observando y el objetivo del análisis, ya que puede referirse desde un canal de irrigación a megaproyectos hidráulicos que interconectan cuencas hidrográficas. La durabilidad, por otro lado, se refiere a la fortaleza y estabilidad entre los elementos heterogéneos que conforman la red, considerando los vínculos y decisiones entre actores humanos y no humanos que son parte de ella. Este atributo también tiene una dimensión temporal -señala Bolding-, ya que la estabilidad del sistema define la permanencia de la red a lo largo del tiempo, es decir, cuánto tiempo dura la red sin que se desmorone. Estas dos características nos permiten analizar las redes hidrosociales que conforman los camiones aljibe que recorren la provincia de Petorca.

\section{Escasez hídrica en Petorca y procesos administrativos para el abastecimiento de agua potable en camiones aljibe}

La provincia de Petorca ha sido, durante la última década, el territorio emblemático de la crisis hídrica en la zona central de Chile. Debido a la megasequía de los últimos diez años, se registran déficits de más del 300\% en comparación con la década anterior. Esta disminución de agua disponible está asociada a la merma de las precipitaciones en un $20 \%$ durante este periodo, y a la sobreexplotación de las fuentes de agua tanto superficiales como subterráneas por efecto de la fuerte expansión agrícola que ha experimentado la zona desde la década de 1990 (Muñoz et al., 2020). La historia reciente de la provincia está marcada por la influencia de las políticas de Estado, que van desde la reforma agraria de la década de 1960, a las políticas neoliberales que permanecen hasta la actualidad (Budds, 2020). Lo anterior se traduce en el fomento de la actividad agroexportadora intensiva en grandes extensiones del territorio que consideran, incluso, laderas de cerro de secano (Panez-Pinto et al., 2017).

En este contexto de explotación agropecuaria y de sequía prolongada, es importante destacar el rol que ha tenido el proceso de comodificación del agua en el territorio, evidenciado por la acumulación de DAA donde, durante el periodo 20002017, tan solo el 4\% de los DAA subterráneas registrados en la provincia son inscritos por empresas sanitarias y comunidades de APR. Así, en la provincia de Petorca, estas tres situaciones condicionantes de la escasez hídrica socialmente producida determinan una orientación del uso del agua hacia las actividades productivas por sobre una priorización del consumo humano y la subsistencia de las comunidades, siendo 
esto último cubierto por camiones aljibe, donde se estima que para el 2014 un tercio de la población de la provincia fue abastecida de esta forma (19.465 personas) (Panez-Pinto et al., 2017).

Dada la importancia que ha tenido este sistema de abastecimiento para la provincia, es de interés conocer las estructuras institucionales diseñadas para establecer su entrega, considerando el sistema burocrático involucrado, los volúmenes de agua repartidos, la distribución espacial de las localidades proveedoras y abastecidas, los costos económicos asociados y los particulares proveedores de esta red.

\section{Procedimiento administrativo para la compra y distribución de agua por camiones aljibe}

La compra y distribución de agua potable por camiones aljibe como medida de emergencia se tramita por medio de dos vías principales: se puede realizar con recursos municipales; y con fondos de emergencia del Ministerio del Interior, a través de la Gobernación Provincial. Nuestro análisis se centra en esta segunda vía, ya que el agua repartida a través de la Gobernación representó el 95\% de los metros cúbicos entregados y el $98 \%$ del gasto público en camiones aljibe en la provincia durante el periodo comprendido entre los ańos 2012 y 2018, ambos inclusive.

El proceso de solicitud de agua mediante la Gobernación Provincial consta de tres etapas administrativas (solicitud, ejecución y rendición), esquematizadas en la Figura 1, que actúan como "llaves de paso" del flujo dentro de la red hidrosocial producida y sostenida por la escasez hídrica. La primera etapa de solicitud se inicia con el aviso de falta de agua a los municipios, o directamente a la Gobernación, por parte de las cooperativas o comités de APR (paso $\mathrm{n}^{\circ} 1$ ). En respuesta, funcionarios municipales visitan el sector y catastran a la población afectada usando la Ficha Básica de Emergencia (FIBE) (paso ${ }^{\circ} 2$ ), como indica la Subsecretaría del Interior (2016). Con base en el catastro de la encuesta FIBE, los municipios deben calcular la cantidad de agua necesaria para satisfacer la demanda de la población según el Oficio 18.867 del Ministerio del Interior del año 2016, el que indica la entrega máxima de 50 litros diarios por persona. Anteriormente a este, se entregaba un promedio de 100 litros, pero variaba dependiendo de cada caso.

Una vez obtenida la estimación de la demanda de agua, el siguiente paso es la elaboración de un informe llamado Alfa, el cual da cuenta de la situación de emergencia y lleva adjuntas las fichas FIBE, el Registro Único de Usuarios que resume la FIBE, y un oficio del alcalde de cada comuna que solicita la entrega de cierta cantidad de agua con base en los catastros realizados, explica la situación y cita decretos de escasez o emergencia (paso $n^{\circ} 3$ ). Todos estos documentos son entregados trimestralmente a la Gobernación Provincial, la cual, a su vez, realiza un informe Alfa con los mismos adjuntos, pero a nivel provincial, para ser entregado a la Oficina Nacional de Emergencia del Ministerio del Interior y Seguridad Pública (ONEMI) (paso $\mathrm{n}^{\circ} 4$ ). Esta entidad revisa la documentación, valida las solicitudes de emergencia generando un código de seguimiento en los denominados FEMER (Formularios de Solicitud de Recursos de Emergencia) (paso $\mathrm{n}^{\circ}$ 5) y pide los recursos a la Subsecretaría del Interior, que revisa nuevamente y aprueba el gasto (o no lo hace). 
FIgURA I | Proceso administrativo de suministro de agua potable con fondos de emergencia del Ministerio del Interior

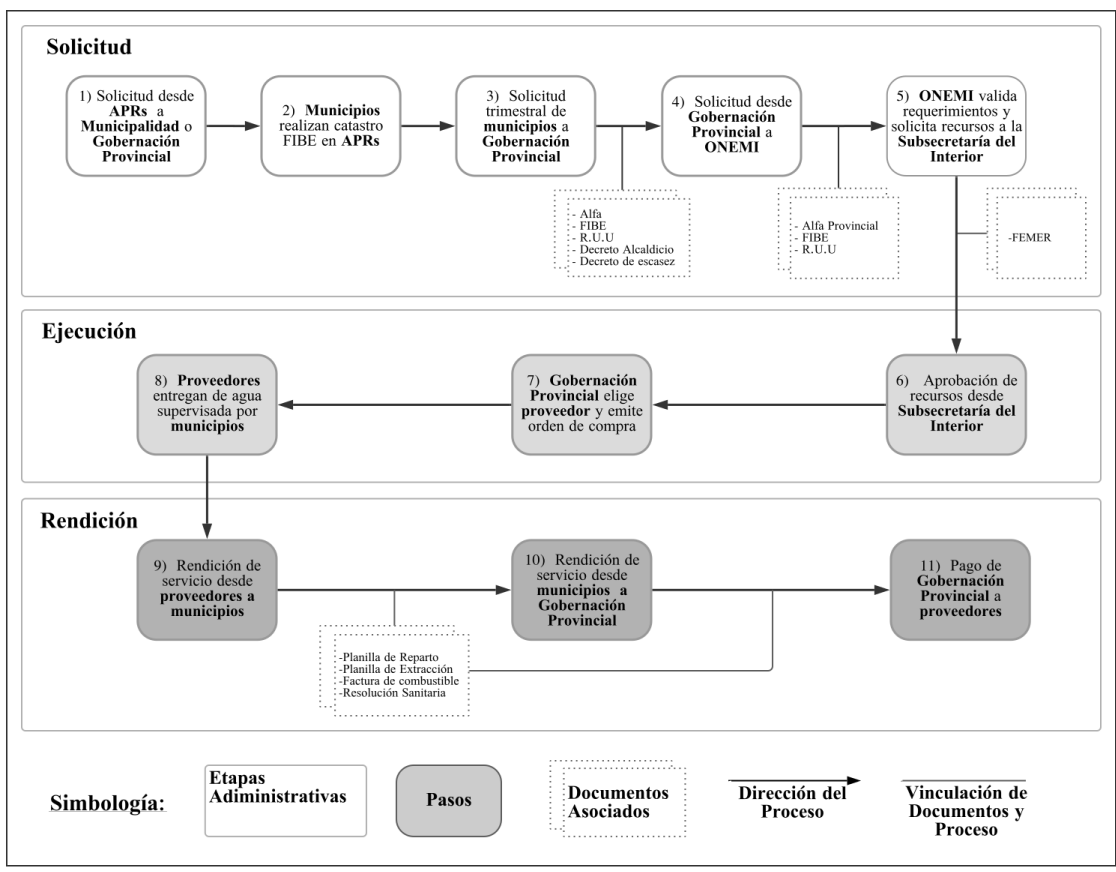

FUENTE: ELABORACIÓN PROPIA CON BASE EN ENTREVISTAS

Una vez aceptada la compra de agua (paso $n^{\circ}$ 6), comienza la etapa de ejecución mediante una orden de compra que emite la Gobernación Provincial, luego de licitar o contratar directamente a proveedores que cuenten con una fuente de extracción de agua con resolución sanitaria (paso $\mathrm{n}^{\circ} 7$ ). De acuerdo con la Coordinadora Regional de Asuntos Hídricos, Subsecretaría del Interior, los contratos se suelen repetir trimestralmente, debido a que es difícil conseguir nuevos proveedores que puedan asumir la tarea con precios competitivos fuera de la zona y que tengan la capacidad de invertir. Estas órdenes, en la mayoría de los casos estudiados, corresponden a la compra conjunta de agua y su transporte, salvo en el caso de las destinadas a abastecer localidades de Petorca y Cabildo, donde solo se licita el agua, debido a que estas municipalidades poseen camiones entregados con recursos de emergencia.

Posteriormente, los municipios coordinan con el proveedor contratado por la Gobernación y fiscalizan el suministro a través de una planilla de reparto, que debe llevar la firma de recepción del encargado en cada comunidad de APR (paso $n^{\circ} 8$ ). Adicionalmente se entrega una planilla de extracción, que registra el día y lugar donde fue obtenida el agua. Estos documentos permiten hacer la rendición del servicio por parte del proveedor a los municipios (paso $n^{\circ}$ 9) y de estos a la Gobernación Provincial (paso $\mathrm{n}^{\circ} 10$ ), la que paga por la entrega de agua, generalmente luego de un año (paso $\left.{ }^{\circ} 11\right)$. Según la Gobernación Provincial, esta condición restrictiva 
reduce la disponibilidad de proveedores y, por lo tanto, de los puntos de extracción de agua a lo largo de la provincia, lo que perjudica la durabilidad de la red.

Si bien esta red de emergencia abastece a una cantidad importante de población, es fuertemente criticada por parte de funcionarios municipales encargados de gestionar la medida, quienes plantean que los criterios para entregar agua son deficientes, por no satisfacer la diversidad de necesidades del agua a nivel doméstico y porque no logran abastecer a la totalidad de la población afectada, dada la dispersión de las localidades de cada comuna. Debido a lo anterior, cada municipio enfrenta la situación de acuerdo con sus capacidades e intereses; en el caso de la Municipalidad de Petorca, se creó la Oficina de Asuntos Hídricos, la cual se encarga particularmente de gestionar y atender problemáticas vinculadas con el agua. Esta oficina ha fomentado y coordinado redes de apoyo entre las distintas comunidades de APR para responder a la falta de agua en las localidades y, a su vez, se encarga de la solicitud de camiones aljibe, como veremos a continuación.

\section{Las dinámicas de distribución de agua potable por camiones aljibe: análisis de los flujos de agua y dinero en la provincia de Petorca}

A continuación, analizamos los resultados de más de 1.700 solicitudes de provisión de agua mediante camiones aljibe que se realizaron para la provincia de Petorca entre los años 2012 y 2018. Empezamos con un análisis volumétrico de los flujos de agua movilizados mediante camiones aljibe, para seguir con su análisis territorial, donde destacamos los sectores que son abastecedores y perceptores de agua. Concluimos analizando los dueńos y el precio del agua distribuida, con el fin de comprender la conformación de la red hidrosocial compuesta por camiones aljibe en la provincia de Petorca.

La distribución de agua potable para consumo humano mediante camiones aljibe en la provincia de Petorca es común desde el año 2008, pero no se hizo masiva hasta el inicio de la megasequía el 2010. Nuestro análisis empieza en 2012, año desde el cual se encuentran registros públicos de las compras de este servicio, y termina el ańo más reciente en que se tenían datos completos, el 2018. Este periodo de estudio logra reflejar el comportamiento general de este modo de abastecimiento de agua durante la megasequía, captando un auge durante los años 2015 y 2016, y su establecimiento como solución al problema de escasez entre comunidades rurales de la provincia a partir de este peak.

Durante el periodo de estudio, las operaciones de la Gobernación para la compra de agua potable y la contratación de su reparto en camiones aljibe en la provincia de Petorca, movilizaron en total $1.494 .492 \mathrm{~m}^{3}$ de agua, que implicaron un gasto total de 8.754 millones de pesos $(\$ 8.754 .121 .511)$, para abastecer a 41 localidades en distintas ventanas de tiempo. La Figura 2 ilustra la evolución temporal tanto del volumen de agua (Figura 2a) como del gasto total (Figura 2b) por comuna. Como se puede apreciar, el gasto monetario y los volúmenes de agua distribuidos tienen la misma tendencia, con un peak en los años 2015 y 2016.

La reducción del volumen de agua repartida a partir del año 2016 (Figura 2a), se puede atribuir parcialmente a la reducción del volumen distribuido por persona 
a partir de este año, por lo que no necesariamente significa una disminución de la necesidad de agua de emergencia. Según el Oficio Ordinario 18.867 del Ministerio del Interior y Seguridad Pública, la entrega de agua mediante camiones aljibe se limita ese año a 50 litros de agua potable diarios por persona, mucho menos que los 100 o 150 litros considerados anteriormente; esta reducción coincide con el abastecimiento mínimo para la sobrevivencia humana (oMs, 2003), limitando la posibilidad de utilizar agua para usos que no sean su consumo directo, invisibilizando así la real necesidad de la población abastecida. Para poder dimensionar la cantidad de agua distribuida en Petorca, cabe mencionar que el promedio nacional de consumo de agua doméstica es igual a 170 litros de agua diarios. ${ }^{2}$

FIGURA 2 | Evolución temporal de distribución de agua mediante camiones aljibe en la provincia de Petorca entre los ańos 2012 y 2018. 2a) Volumen de agua entregada por la Gobernación Provincial en cada comuna en $\mathrm{m}^{3} / \mathrm{ańno.} \mathrm{2b)} \mathrm{Gasto} \mathrm{en} \mathrm{pesos} \mathrm{de} \mathrm{la} \mathrm{Gobernación} \mathrm{Provincial}$ por comuna en pesos/año

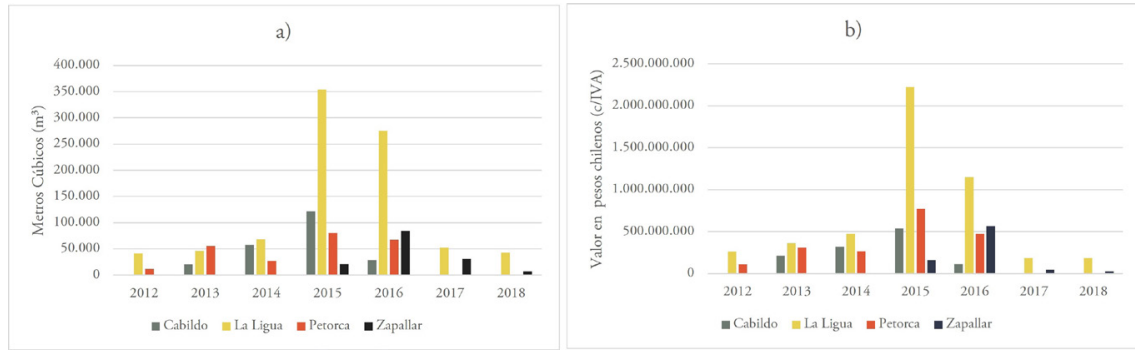

FUENTE: ELABORACIÓN PROPIA BASADA EN ÓRDENES DE COMPRA EN EL PERIODO 2OI 2-2OI 8

Estos flujos de agua potable movilizada por camiones no solo se caracterizan por su volumen y precio, también por su trayectoria: el agua potable se carga a los camiones en sus puntos de extracción y se descarga en los lugares necesitados. Las órdenes de compra, realizadas según el procedimiento descrito anteriormente, son licitadas o contratadas directamente a proveedores en la provincia, ${ }^{3}$ quienes entregan agua subterránea extraída desde sus pozos particulares a comités o cooperativas de APR. Los trayectos de los camiones aljibe, según el análisis de las órdenes de compra entre los ańos 2012 y 2018, son cortos, puesto que en unos casos el agua se extrae y entrega dentro del mismo sHac (Figura 3).

2 El consumo de agua promedio por persona en Chile supera la media de Europa, aunque está muy por debajo de la de Estados Unidos (El Mercurio, 2020).

3 De los datos analizados, se desprende que el $83 \%$ de los proveedores extraen agua dentro de la provincia, mientras que se desconoce el punto de captación del 17\% restante. 
FIGURA 3 | Divisiones político-administrativas comunales y Sectores Hidrogeológicos de Aprovechamiento Común (sHAC) definidos por la Dirección General de Aguas (DGA)

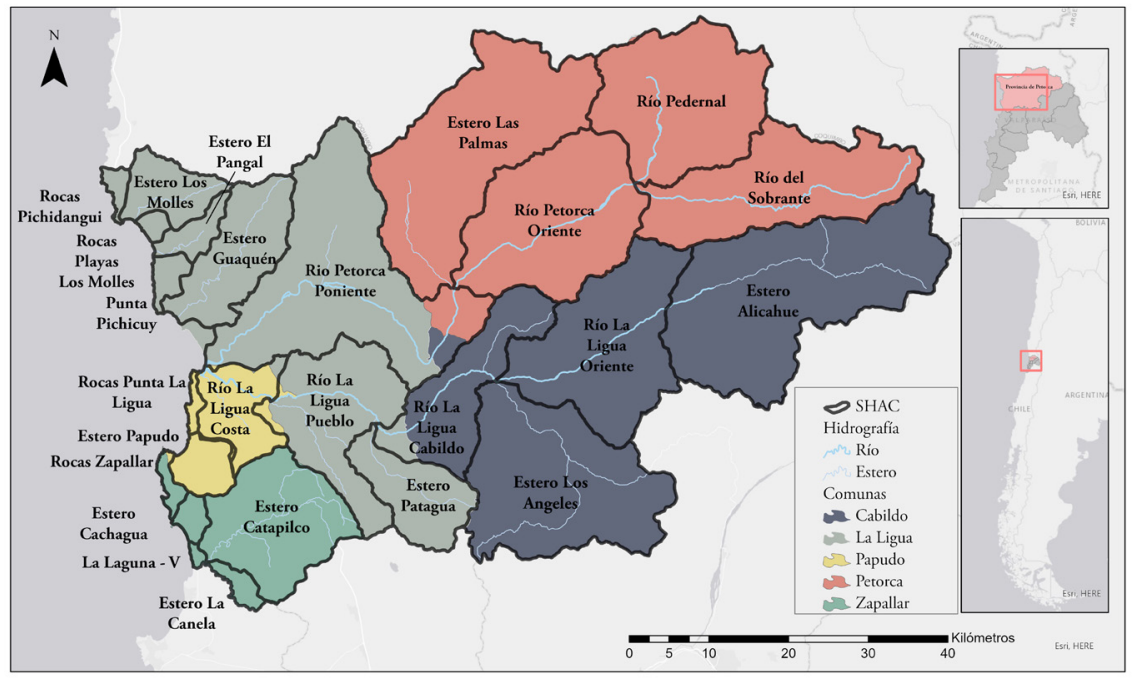

FUENTE: ELABORACIÓN PROPIA CON BASE EN DATOS DE LA BIBLIOTECA DEL CONGRESO NACIONAL (BCN) Y DGA

FIGURA 4 | Ubicación de los pozos de extracción de agua inscritos por los proveedores, las localidades con APR abastecidas por camiones $y$ sus SHAC correspondientes durante el periodo 2012-2018

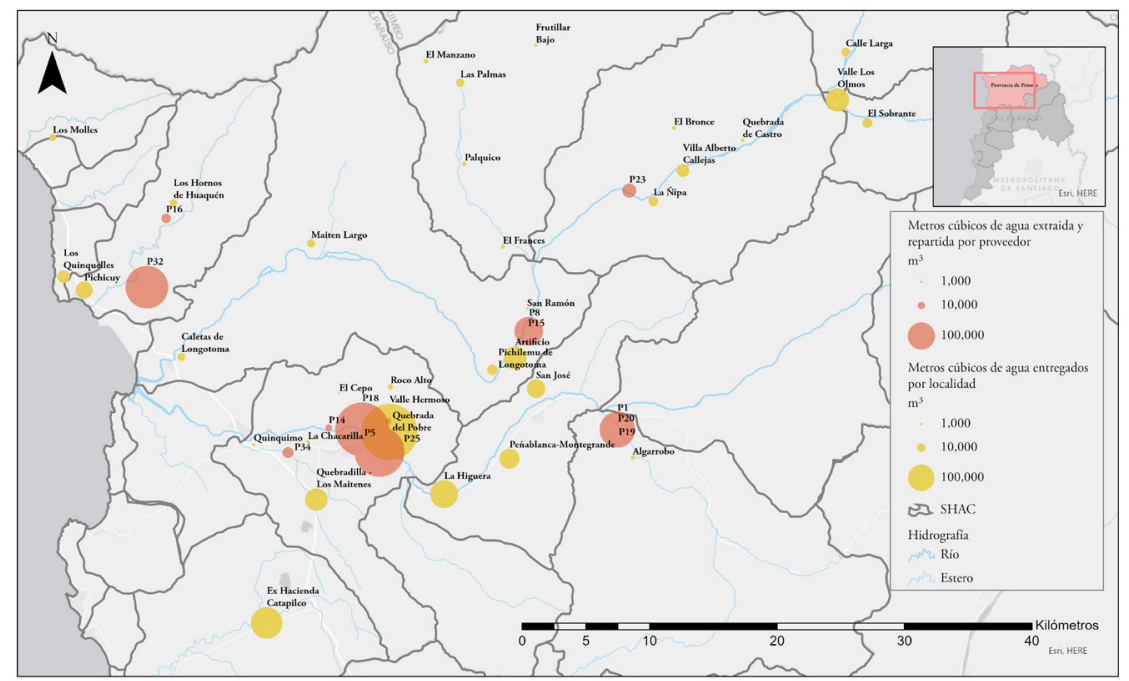

FUENTE: ELABORACIÓN PROPIA A PARTIR DE ÓRDENES DE COMPRA DE AGUA Y REGISTRO PÚBLICO DE DERECHOS DE APROVECHAMIENTO DE AGUAS DEL CONSERVADOR DE BIENES RAÍCES 
Durante el período de estudio, el agua distribuida por camiones aljibe fue proporcionada por 23 proveedores que extrajeron agua de cinco distintos SHAC y la distribuyeron dentro de estos mismos y en otros seis en la provincia. Destaca especialmente el sHAC "Ligua Pueblo" (Figura 4 y Figura 5), como aquel que proporciona la mayor cantidad de agua para su distribución por camiones aljibe mediante dos proveedores $\left(670.240 \mathrm{~m}^{3}\right)$ y es, al mismo tiempo, el sector que mayor cantidad de agua ha recibido $\left(546.910 \mathrm{~m}^{3}\right), 42 \%$ de la cual es proveniente del mismo sector. En este sHaC se encuentra circunscrita la localidad de Valle Hermoso, que ha sido abastecida durante el periodo de estudio por un total de $458.053 \mathrm{~m}^{3}$ y que destaca por encontrarse a pocos kilómetros de sus proveedores de agua por camiones aljibe (Figura 5). Esta situación evidencia la dimensión social de la escasez hídrica en la zona, puesto que indica que más que una falta física de agua en las cuencas de los ríos Petorca y La Ligua, existe una inequidad distributiva de los DAA que afecta a los comités y cooperativas de APR, y que se agrava con la insuficiente infraestructura al servicio de estas comunidades (Fundación Amulén et al., 2019; Oppliger et al., 2019). Otra de las localidades con mayor volumen de abastecimiento es la APR ex Hacienda de Catapilco, con un total de $142.665 \mathrm{~m}^{3}$ durante el periodo de estudio. Ambas localidades corresponden a sectores de expansión urbana con alta densidad poblacional en relación con el resto de las comunidades de APR; sin embargo, no se encuentran dentro de la zona de cobertura para zonas urbanas que entrega la empresa sanitaria regional ESVAL.

FIGURA 5 | Flujos de agua potable por camiones aljibe entre SHAC, periodo 20122018. Diagrama Sankey. A la izquierda se grafican los sHAC de origen, con su respectivo nombre y tamańo proporcional al porcentaje que representan; al centro se muestran los proveedores que venden el agua; y a la derecha, los SHAC de destino que contienen las localidades abastecidas

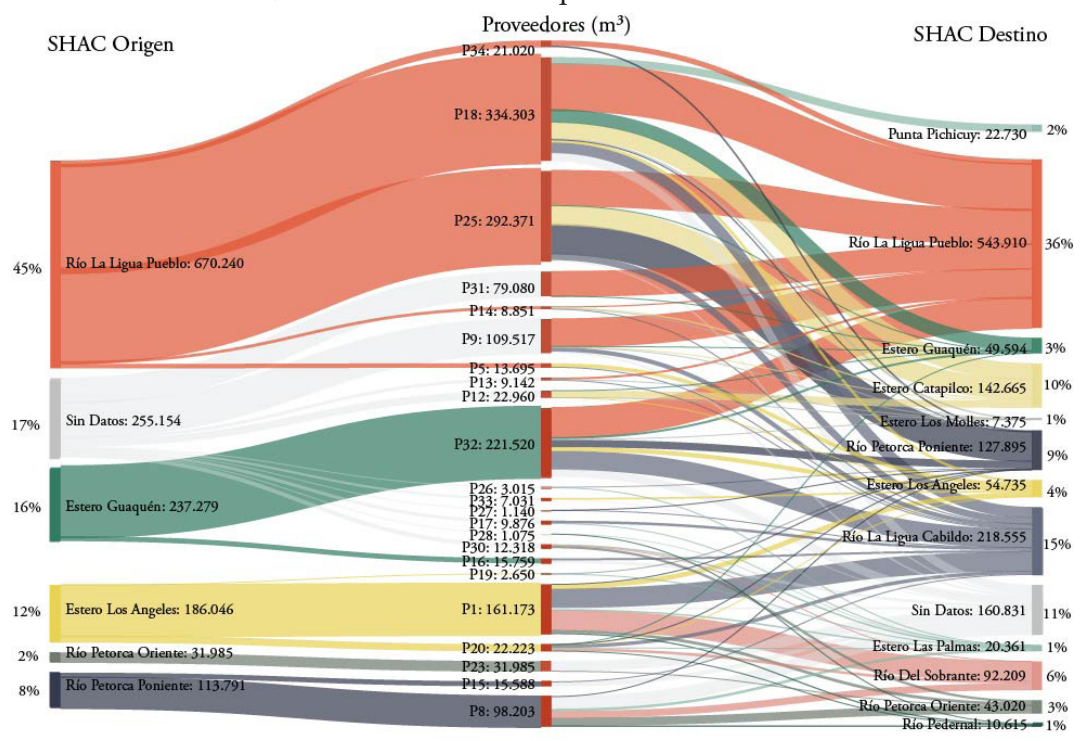

FUENTE: ELABORACIÓN PROPIA A PARTIR DE ÓRDENES DE COMPRA DE AGUA EN REGISTRO PÚBLICO DE DERECHOS DE APROVECHAMiENTOS DE AGUAS (hTTPS://DGA.MOP.GOB.Cl/) Y CATASTRO DEL CONSERVADOR DE BIENES RAÍCES (HTTPS://WWW.CONSERVADOR.CL/PORTAL/GP_AGUAS) 
Por otra parte, según las entrevistas realizadas para la investigación, las localidades que han recibido menos volumen de agua a través de este mecanismo corresponden a sistemas de comunidades de APR que abastecen a poca población, pero que padecen de igual o peor forma la escasez hídrica, debido a que administrativamente cuesta más conseguir recursos para mejora de infraestructura hidráulica, por su poca rentabilidad social.

Las diferencias entre sectores no se restringen a los que proveen agua y los que la reciben; es importante considerar que el precio del agua potable por metro cúbico entregada de esta forma varía notablemente entre localidades (Tabla 1). Esto se explica principalmente por el costo de transporte del agua, que incluye combustible, horas de trabajo de los operarios y la frecuencia de recarga de los camiones. Este factor está incluido sin especificación en el monto de las órdenes de compra estudiadas y aumenta significativamente el gasto en la entrega de este recurso a localidades más alejadas de sus proveedores, como lo son El Maray, Frutillar Bajo, Quebrada de Castro, Valle Los Olmos y El Manzano (Figura 4).

El precio del agua distribuida por camiones aljibe es claramente más alto que el agua distribuida por las comunidades de APR locales. Por ejemplo, un hogar de cuatro personas que consume 100 litros diarios por persona consumiría en total $12 \mathrm{~m}^{3} /$ mes, lo que costaría entre 6.000 y 8.000 pesos en las comunidades APR de Petorca bajo condiciones normales de disponibilidad hídrica; si el mismo hogar se abasteciera de agua con camiones aljibe, este mismo volumen le costaría más de 32.000 pesos. A pesar de que este gasto no es a cargo directo de los consumidores, estos datos indican el despilfarro de recursos económicos que ha implicado esta solución para el Estado y las autoridades regionales, como también la importancia de este mercado que surge a partir de la sequía.

En lo que respecta a los dueños del agua que se distribuye mediante camiones aljibe, la Figura 6 presenta la concentración de dinero obtenido por los proveedores, según las órdenes de compra. Los datos demuestran que, si bien son más de veinte empresas o personas naturales las que abastecen a los camiones con agua, cerca del $80 \%$ del gasto se concentra en apenas cinco proveedores, cuatro de los cuales recibieron más de mil millones de pesos durante el periodo 2012-2018. 
TABLA I | Volumen, promedio precio por metro cúbico y valor del agua distribuida por camiones aljibe en las localidades de las cuatro comunas de la provincia de Petorca entre los ańos 2012 y 2018

\begin{tabular}{|c|c|c|c|c|}
\hline COMUNA & $\begin{array}{c}\text { AGUA POTABLE RURAL } \\
\text { (APR) }\end{array}$ & $\mathrm{M}^{3}$ TOTALES & $\begin{array}{c}\text { PRECIO } \\
\text { PROMEDIO M } \\
\text { (PESOS CHILENOS) }\end{array}$ & $\begin{array}{c}\text { PRECIO DE } \mathrm{M}^{3} \\
\text { TOTALES (C/IVA) } \\
\text { (PESOS CHILENOS) }\end{array}$ \\
\hline \multirow{7}{*}{ Cabildo } & Algarrobo & 2.840 & 4.500 & 15.208 .200 \\
\hline & El Maray & 500 & 2.500 & 14.875 .000 \\
\hline & No especifica & 12.884 & 7.219 & 104.803 .300 \\
\hline & San José & 48.795 & 3.823 & 218.017 .520 \\
\hline & Peńablanca-Montegrande & 57.735 & 3.780 & 249.898 .965 \\
\hline & Bartolillo Paihuén & 29.191 & 7.725 & 251.631 .450 \\
\hline & Artificio & 75.980 & 3.572 & 324.780 .750 \\
\hline \multirow{14}{*}{ Petorca } & San Ramón & 935 & 6.402 & 7.032 .900 \\
\hline & El Francés & 2.861 & 7.512 & 16.833 .835 \\
\hline & El Bronce & 2.915 & 5.541 & 19.487 .440 \\
\hline & Frutillar Bajo & 1.320 & 17.697 & 27.798 .311 \\
\hline & Quebrada de Castro & 1.881 & 14.936 & 33.740 .013 \\
\hline & Palquico & 3.029 & 10.839 & 39.129 .541 \\
\hline & El Manzano & 3.223 & 17.310 & 66.466 .417 \\
\hline & La Nipa & 13.586 & 5.320 & 87.450 .720 \\
\hline & Las Palmas & 9.928 & 10.871 & 128.732 .124 \\
\hline & Villa Alberto Callejas & 23.670 & 4.636 & 130.562 .040 \\
\hline & Calle Larga & 10.615 & 13.082 & 169.996 .855 \\
\hline & El Sobrante & 14.820 & 9.897 & 179.856 .600 \\
\hline & No especifica & 76.251 & 5.072 & 428.777 .825 \\
\hline & Valle Los Olmos & 77.389 & 11.444 & 597.496 .620 \\
\hline Zapallar & Ex Hacienda Catapilco & 142.665 & 5.071 & 800.709 .149 \\
\hline \multirow{22}{*}{ La Ligua } & Guindo Chico & 200 & 7.560 & 1.799 .280 \\
\hline & El Cepo & 754 & 3.100 & 2.921 .450 \\
\hline & La Chacarilla & 1.750 & 3.656 & 7.840 .028 \\
\hline & Quinquimo & 1.589 & 4.872 & 9.737 .050 \\
\hline & Roco Alto & 4.190 & 3.420 & 12.852 .000 \\
\hline & $\begin{array}{l}\text { Pichilemu y APR La } \\
\text { Higuera }\end{array}$ & 4.250 & 4.560 & 22.704 .681 \\
\hline & $\begin{array}{l}\text { Quebrada del Pobre (Paso } \\
\text { Oscuro) }\end{array}$ & 5.240 & 4.930 & 29.030 .764 \\
\hline & Pullancon Los Romeros & 4.590 & 13.600 & 71.602 .300 \\
\hline & El Guayacán & 5.374 & 11.727 & 80.681 .762 \\
\hline & Los Molles & 7.375 & 13.761 & 92.931 .754 \\
\hline & Los Hornos de Huaquén & 8.004 & 10.971 & 108.750 .292 \\
\hline & Caletas de Longotoma & 9.100 & 10.170 & 110.471 .318 \\
\hline & Maitén Largo & 9.460 & 11.100 & 125.848 .450 \\
\hline & Pichilemu de Longotoma & 15.500 & 10.610 & 204.840 .174 \\
\hline & La Canela & 12.130 & 14.760 & 214.934 .944 \\
\hline & San Lorenzo (Casas Viejas) & 17.330 & 11.717 & 236.334 .000 \\
\hline & Quebradilla-Los Maitenes & 72.384 & 4.773 & 245.170 .252 \\
\hline & Los Quinquelles & 22.730 & 11.716 & 316.175 .828 \\
\hline & La Higuera & 111.975 & 3.363 & 451.478 .131 \\
\hline & No especifica & 67.913 & 7.099 & 501.161 .693 \\
\hline & Pichicuy & 41.590 & 11.541 & 527.661 .232 \\
\hline & Valle Hermoso & 458.053 & 2.729 & 1.465 .908 .554 \\
\hline \multicolumn{2}{|l|}{ TOTAL } & 1.494 .494 & 8.079 & 8.754 .121 .512 \\
\hline
\end{tabular}

FUENTE: ELABORACIÓN PROPIA A PARTIR DE ÓRDENES DE COMPRA DE AGUA 
FIGURA 6 | Gasto c/IVA por proveedor, Gobernación Provincial 2012-2018

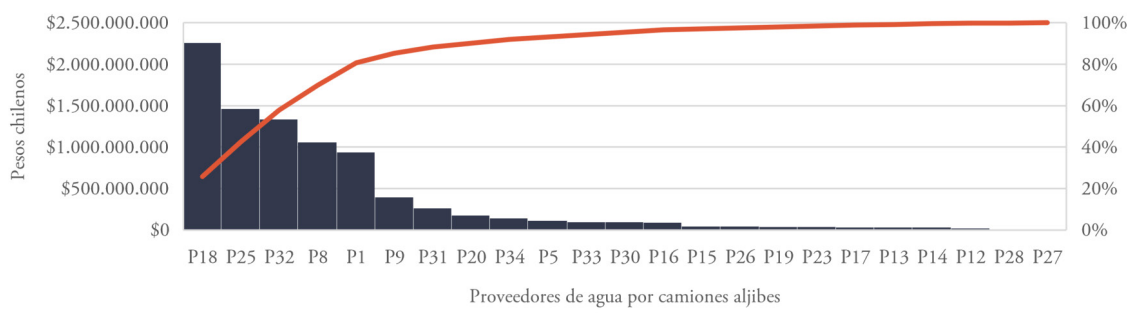

FUENTE: ELABORACIÓN PROPIA A PARTIR DE ÓRDENES DE COMPRA DE AGUA

Al enfocarnos en los cinco proveedores principales y sus DAA inscritos en la zona, fue posible identificar que al menos tres de ellos tienen derechos inscritos para uso agrícola. Además, cada uno de ellos compró DAA durante el periodo estudiado. Es así como una empresa agrícola (P1) adquirió 7 litros por segundo (l/s) entre los ańos 2013 y 2016, mientras que, en lo que respecta a personas naturales, P18 adquirió 6,8 l/s entre 2014 y 2017; P8, 3,65 l/s entre 2014 y 2015; P25, 2 l/s aproximadamente en 2016; y P32, 1,15 l/s el 2015. Esta situación se vuelve relevante al compararla con las cantidades de agua entregada por estos actores, lo que es apreciable en la Figura 7, donde se muestran los metros cúbicos entregados por los cinco proveedores en cada ańo estudiado. Estos resultados indican que la venta de agua potable mediante camiones aljibe es una actividad rentable y dominada por pocos actores, y con una fuerte participación de empresas y particulares del sector agrícola, que es otra manifestación de la escasez hídrica de causas sociales en la provincia de Petorca.

\section{FIGURA 7 | Agua vendida por camiones por principales proveedores y DAA}

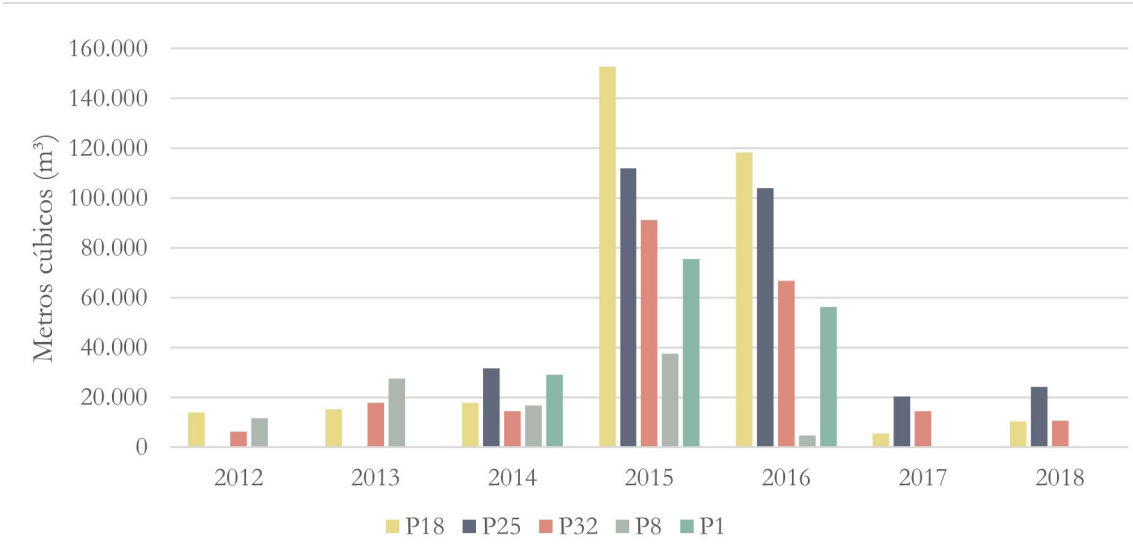

FUENTE: ELABORACIÓN PROPIA A PARTIR DE ÓRDENES DE COMPRA DE AGUA 


\section{Los camiones aljibe como red hidrosocial: alcance y durabilidad de un sistema de emergencia}

La expresión en el territorio de la red hidrosocial de camiones aljibe en Petorca permite, por una parte, identificar los elementos que la componen (camiones aljibe, el volumen transportado, sus proveedores y consumidores); y, por otra parte, transparenta cómo se decide la circulación de agua potable de emergencia a comunidades rurales en la provincia, siguiendo un esquema burocrático de escalas que superan lo local y que abordan el problema de la escasez hídrica como consecuencia de un evento climático extremo. Así, los resultados obtenidos dan cuenta de la consolidación de un aparato estatal que transforma esta respuesta reactiva, de emergencia y temporal, en un mecanismo establecido en el territorio que se incorpora a la vida cotidiana de las comunidades que lo habitan y profundiza sus condiciones de escasez hídrica.

Utilizando los atributos de la red hidrosocial, identificamos que su alcance cubre dispersas localidades rurales en la provincia, ubicadas desde la costa hasta la sección alta de la cuenca del río Petorca, donde los puntos de captación de agua potable y las localidades receptoras se concentran en la sección baja de la cuenca del río La Ligua. Además, al observar los flujos hídricos entre los sectores hidrogeológicos, particularmente en el sector de Valle Hermoso (shac Ligua-Pueblo), el alcance de la red nos permite identificar un hallazgo relevante: el mayor volumen de aguas movilizadas abastece al mismo sector del cual fueron extraídas, es decir, el origen y destino de las aguas están ubicados en el mismo sector hidrogeológico. El reducido alcance de la red, manifestado por la extracción y distribución de agua dentro de un mismo SHAC, evidencia que no todos los actores se ven impactados de la misma manera por la sequía. Mientras que las comunidades de APR necesitan de agua potable por medios de emergencia, hay empresas y personas naturales, principalmente del rubro agrícola, que tienen un superávit hídrico que pueden distribuir en el mercado de venta de agua por camiones aljibe.

Por otro lado, dado que esta red se trata -formal e institucionalmente- como de "emergencia", los actores involucrados van desde las comunidades de APR hasta la Subsecretaría del Interior, pasando por los municipios, la gobernación provincial y la ONEMI. La burocracia en el proceso, el número de instituciones involucradas y la fuerte carga administrativa de los municipios, genera que el alcance de esta medida no logre satisfacer la real demanda de agua potable de otras localidades que no están siendo consideradas en los catastros oficiales y, por tanto, se ven obligadas a encontrar otras formas precarias de abastecimiento. Además, el instrumento aplicado para reconocer la falta de agua de la población afectada (ficha FIBE) proveía de 50 litros a cada persona el 2016, considerado por la oms como la cantidad mínima necesaria para la sobrevivencia humana (Howard \& Bartram, 2003); y, por tanto, resulta insuficiente para mantener los estilos de vida de las distintas comunidades, perjudicando la vida rural chilena. Es decir, el alcance de la medida no logra transmitir la gravedad del estrés hídrico en el territorio ni apoyar a todas las comunidades afectadas, presionándolas a encontrar otras alternativas que les permitan subsistir en la provincia. 
Según esto, una de las características del alcance de la red hidrosocial es su variabilidad, ya que requiere de grandes esfuerzos para ejecutar y registrar el problema en todas las localidades sin agua (fichas FIBE), como también de arreglos institucionales que pueden modificar el volumen de agua que debe ser entregado por persona en contextos de emergencia.

En términos de durabilidad de la red hidrosocial, ella se puede analizar en dos escalas. En la primera, donde se observa cada localidad de manera individual, identificamos que la durabilidad es corta en términos formales, puesto que está definida para que no se extienda más allá de los seis meses que permite la emisión de un decreto de escasez. En conjunto con los engorrosos y lentos trámites burocráticos que se deben realizar para la solicitud, ejecución y rendición de la medida, esto resulta en un intermitente abastecimiento para las localidades impactadas por la escasez. Sin embargo, al observar la durabilidad de esta medida en una escala provincial, la red de abastecimiento conformada por los camiones aljibe tiene larga durabilidad, puesto que al menos desde el 2012 se ha perpetuado la ejecución de esta medida para distintas comunidades en la provincia. En otras palabras, la durabilidad de la red es aperiódica para las localidades, pero permanece en el tiempo para la provincia en su conjunto, a pesar de ser una medida de abastecimiento de emergencia.

Además, el rol del Código de Aguas les otorga estabilidad a los proveedores, ya que permite que los cinco principales proveedores de agua en esta red hidrosocial, que concentran más del $80 \%$ de las ganancias del periodo de estudio, incrementen su dominio de derechos de aprovechamiento de agua en los años analizados, pudiendo aumentar sus ingresos en escenarios de escasez hídrica. Al profundizar en quiénes son estos proveedores principales, sobresale que tres de los cinco proveedores sean del sector agrícola y concentren el $43 \%$ de los ingresos totales durante el periodo. Si bien en este estudio no se profundizó en el rol del sector agrícola en la provincia, la evidencia permite demostrar que es el sector económico que ha generado la escasez en la provincia, dada la agroexportación (Panez-Pinto et al., 2017); y que, además, ha podido vender y distribuir el agua potable a quienes no tienen los medios para acceder a ella.

\section{Conclusiones}

En este artículo presentamos el inédito estudio de la distribución de agua potable mediante camiones aljibe durante la megasequía en la provincia de Petorca, una de las zonas más afectadas por este fenómeno. Mediante análisis volumétricos y espaciales, basados en las órdenes públicas de compra, detectamos por primera vez los flujos de agua que se extraen, transportan y consumen en Petorca mediante este sistema, considerando su precio, sus vendedores y sus consumidores. Una segunda contribución del trabajo es la propuesta de analizar este sistema de abastecimiento de agua como una red hidrosocial, un concepto que capta la complejidad y multiescalaridad de modos de abastecimiento de agua potable que escapan de la categoría de infraestructura hidráulica, por su carácter efímero y de emergencia. Esto es de especial relevancia, dada la importancia que tienen estas estructuras efímeras para 
abastecer con agua a comunidades rurales en el Sur global, en un contexto de cambios climáticos y de disminución de fuentes tradicionales de agua fresca.

Las redes de abastecimiento hídrico conformadas por camiones aljibe se basan en un mecanismo burocrático que implica un alto y constante esfuerzo administrativo, económico y operacional que no corresponde a una medida de emergencia que entrega un recurso vital. Al mismo tiempo, no logra satisfacer la demanda total de las localidades y cubrir el déficit hídrico de los usos afectados, ya que el consumo promedio repartido por persona ha llegado a ser 50 litros por persona al día, apenas el 30\% del consumo promedio nacional de 170 litros por persona diario. Por otro lado, la instalación de este sistema de abastecimiento desplaza la urgencia de desarrollar soluciones para destrabar la crisis a largo plazo, mientras instala un mercado muy rentable para los principales aguatenientes de la zona. El hecho de que este mercado esté dominado por unos pocos actores locales, principalmente del rubro agrícola, que venden millones de metros cúbicos de agua anualmente para su consumo en comunidades de APR vecinas, apoya el argumento de que en Petorca no existe una escasez física y absoluta, sino una desigual distribución del agua.

Los altos volúmenes de agua vendida y las compras de nuevos DAA por los principales vendedores de agua durante la sequía dan evidencia, además, de la alta rentabilidad de este negocio, donde se prefiere destinar el agua para su venta para uso doméstico en vez de agrícola. Además, la desigualdad estructural no permite que las comunidades de APR puedan adquirir DAA; y, en el caso de contar con ellos, tampoco disponen de la infraestructura ni de los artefactos que permitan extraerla. En ese sentido, los altos montos de dinero que ha desembolsado el Estado para la compra de agua podrían haber sido destinados a la ayuda estructural a las comunidades de APR locales con medidas como: i) el aseguramiento de la cantidad de agua necesaria por persona (calculada con base en las necesidades locales), mediante la expropiación o compra de derechos de agua para las comunidades de APR, la priorización de usos y la efectiva eliminación del lucro con los DAA; ii) mejoras infraestructurales, técnicas, operacionales y de gestión de las comunidades de APR para garantizar su sustentabilidad; y iii) el fomento de la asociatividad y colaboración entre las mismas comunidades de APR, algo que está demostrado que aumenta la resiliencia de los sistemas socioecológicos.

Más allá de los análisis tangibles y cuantitativos, el estudio de la red de abastecimiento de agua con camiones aljibe como una red hidrosocial a partir de su alcance y durabilidad nos revela otras características de ella. El alcance de la red, entendida como su alcance espacial, social, material e institucional, es variante, ya que se expande y recoge espacialmente según las necesidades hídricas de las comunidades de APR, implicando la dificultad de establecer y delimitar la red. Si consideramos que el agua entregada no es suficiente para satisfacer las necesidades de la población, podríamos decir que la red no tiene el mismo alcance material que espacial, ya que logra llegar donde se solicita, pero no se entrega el volumen suficiente. Con respecto al alcance social, la red se amplía a comunidades normalmente marginalizadas geográficamente y de bajo nivel socioeconómico; en términos institucionales, se expande a muchos niveles y varias instituciones, y es sostenida en procesos lentos y engorrosos. Todas estas características apuntan a la precariedad de la red. 
Con respecto a la durabilidad -un atributo que se refiere a la fortaleza y estabilidad entre los elementos heterogéneos que conforman la red y tiene una dimensión temporal-, podemos decir que es ambigua. Por un lado, y a pesar de ser una medida de emergencia, el sistema de camiones aljibe no solo se ha mantenido, sino que se ha establecido como modo de abastecimiento de agua potable en zonas rurales con escasez hídrica, indicando una larga durabilidad temporal en su conjunto, a pesar de su cambiante alcance espacial. Por otro lado, la debilidad de las comunidades de APR y la fragilidad de la red de abastecimiento indican baja fortaleza de la red; esta depende de diversa infraestructura (camiones, petróleo, caminos) y decisiones momentáneas (decretos), que la hacen sensible a amenazas que alteran su funcionamiento, como el precio del petróleo, el acceso y el fluido movimiento en las calles regionales, la disponibilidad de agua a la venta o problemas de almacenamiento del agua transportada, entre otras.

En total, este análisis nos permite ver la distribución de agua con camiones aljibe como una red secundaria de abastecimiento de agua potable que viene a cumplir los vacíos que dejan las redes de agua "formales" en épocas de escasez hídrica. A pesar de mantenerse estable en el tiempo, la expansión espacial y el volumen de agua entregada por la red cambian según los decretos de escasez emitidos en escalas geográficas y burocráticas muy alejadas del territorio en necesidad hídrica. La red de abastecimiento de agua con camiones aljibe es una estructura lenta, frágil y vulnerable, que depende de un esquema burocrático engorroso y entrega agua demasiado cara para cualquier persona. Vemos nuevamente una solución basada en el mercado, el asistencialismo estatal, y soluciones que nunca fueron consultadas con las comunidades afectadas.

Finalmente, el concepto de red hidrosocial nos permite revelar injusticias hídricas y sociales en términos de la circulación del agua entre los distintos sectores, y la inequidad en el acceso al agua entre las comunidades de APR y los grandes aguatenientes, que incluso pueden vender sus excedentes de agua. A partir de estos hallazgos se hace necesario profundizar en esta temática, replicando este tipo de estudios en otras localidades donde la emergencia hídrica se ha enfrentado mediante el abastecimiento por camiones aljibe, con el fin de entender de manera más integral las dinámicas y problemáticas asociadas a este tipo de abastecimiento. Por otro lado, es imprescindible que se evalúe y garantice la seguridad hídrica que este tipo de abastecimiento hídrico ofrece a nivel doméstico, en términos de la calidad y continuidad del servicio entregado, como también la aceptabilidad de esta agua para su consumo directo, una problemática contemporánea mundial en el marco del cambio climático y su impacto en la disponibilidad hídrica. 


\section{Referencias bibliográficas}

Arellano, A. (2017, marzo 21). El negocio de la sequía: El puñado de empresas de camiones aljibe que se reparte 92 mil millones. CIPER Chile. https://www.ciperchile.cl/2017/03/21/ el-negocio-de-la-sequia-el-punado-de-empresas-de-camiones-aljibe-que-se-reparte92-mil-millones/

Bauer, C. J. (2015). Water conflicts and entrenched governance problems in Chile's market model. Water Alternatives, 8(2), 147-172. https://www.water-alternatives.org/index. php/alldoc/articles/vol8/v8issue2/285-a8-2-8/file

Blomkvist, P., Nilsson, D., Juma, B. \& Sitoki, L. (2020). Bridging the critical interface: Ambidextrous innovation for water provision in Nairobis informal settlements. Technology in Society, 60, 101221. https://doi.org/10.1016/j.techsoc.2019.101221

Boelens, R., Hoogesteger, J., Swyngedouw, E., Vos, J. \& Wester, P. (2016). Hydrosocial territories: A political ecology perspective. Water International, 41(1), 1-14. https:// doi.org/10.1080/02508060.2016.1134898

Bolding, A. (2004). In Hot Water. A study on sociotechnical intervention models and practices of water use in smallholder agriculture, Nyanyadzi catchment, Zimbabwe. Wageningen University and Research, the Netherlands. https://edepot.wur.nl/164902

Bravo, L. \& Fragkou, M. C. (2019). Escasez hídrica, género, y cultura mapuche. Un análisis desde la ecología política feminista. Polis, 18(54), 64-88. https://doi.org/10.32735/ s0718-6568/2019-n54-1401

Broto, V. C., Allen, A. \& Rapoport, E. (2012). Interdisciplinary Perspectives on urban metabolism. Journal of Industrial Ecology, 16(6), 851-861. https://doi.org/10.1111/ j.1530-9290.2012.00556.x

Budds, J. (2009). Contested $\mathrm{H}_{2} \mathrm{O}$ : Science, policy and politics in water resources management in Chile. Geoforum, 40(3), 418-430. https://doi.org/10.1016/j.geoforum.2008.12.008

Budds, J. (2020). Securing the market: Water security and the internal contradictions of Chile's Water Code. Geoforum, 113, 165-175. https://doi.org/10.1016/j. geoforum.2018.09.027

El Mercurio. (2020, febrero 2). Consumo de agua promedio por persona en Chile supera la media de Europa, aunque está muy por debajo de la de Estados Unidos. El Mercurio, cuerpo B, p. 10. https://bit.ly/36LUx8m

Equipo Ameonna. (2020, diciembre 21). 222 mil millones gastó el Estado en contratar camiones aljibe y más de 11 mil millones en comprar forraje para ganado. Vergara 240 [Plataforma digital de la Escuela de Periodismo, Universidad Diego Portales]. https://vergara240.udp.cl/especiales/222-mil-millones-gasto-el-estado-en-contratarcamiones-aljibe-y-mas-de-11-mil-millones-en-comprar-forraje-para-ganado/

Escobar, A. (2008). Territories of difference: Place, movements, life, redes. Duke University Press.

Freire-González, J. \& Puig-Ventosa, I. (2016). Effects of the hydraulic infrastructure on economic growth: Evidence from Catalonia. Sustainable Water Resources Management, 2(2), 199-206. https://doi.org/10.1007/s40899-016-0052-9

Fundación Amulén, Centro de Cambio Global UC \& Centro de Derecho y Gestión de Aguas UC. (2019). Pobres de Agua: Radiografia del agua rural en Chile. https://cambioglobal. uc.cl/proyectos/289-pobres-de-agua-radiografia-del-agua-rural-en-chile 
Hommes, L. \& Boelens, R. (2017). Urbanizing rural waters: Rural-urban water transfers and the reconfiguration of hydrosocial territories in Lima. Political Geography, 57, 71-80. https://doi.org/10.1016/j.polgeo.2016.12.002

Hommes, L., Boelens, R. \& Maat, H. (2016). Contested hydrosocial territories and disputed water governance: Struggles and competing claims over the Ilisu Dam development in southeastern Turkey. Geoforum, 71, 9-20. https://doi.org/10.1016/j. geoforum.2016.02.015

Hoogesteger, J. \& Wester, P. (2015). Intensive groundwater use and (in)equity: Processes and governance challenges. Environmental Science \& Policy, 51, 117-124. https://doi. org/10.1016/j.envsci.2015.04.004

Howard, G. \& Bartram, J. (2003). Domestic water quantity, service level and health. [WHO/ sDE/wsH/03.02]. World Health Organization (wHO). https://www.who.int/water_ sanitation_health/diseases/WSH03.02.pdf?ua=1

Law, J. (1993). Organizing modernity: Social ordering and social theory. Wiley-Blackwell.

Ministerio de Obras Públicas, Chile. (2014). Decreto 203/2014 Aprueba reglamento sobre normas de explotación y exploración de aguas subterráneas. Chile. https://www.bcn.cl/ leychile/navegar?idNorma $=1060095$

Morinville, C. (2017). Sachet water: Regulation and implications for access and equity in Accra, Ghana. WIREs Water, 4(6), e1244. https://doi.org/10.1002/wat2.1244

Muñoz, A. A., Klock-Barría, K., Alvarez-Garreton, C., Aguilera-Betti, I., González-Reyes, Á., Lastra, J. A., Chávez, R. O., Barría, P., Christie, D., Rojas-Badilla, M. \& LeQuesne, C. (2020). Water crisis in Petorca Basin, Chile: The Combined effects of a mega-drought and water management. Water, 12(3), 648. https://doi.org/10.3390/w12030648

Nazer, A., Pavez, O., Zúńiga, B. \& González, L. (2018). Determinación del consumo de agua potable durante la construcción de viviendas en una zona semi desértica de Chile. Matéria, 23(3). https://doi.org/10.1590/s1517-707620180003.0535

Oppliger, A., Höhl, J. \& Fragkou, M. (2019). Escasez de agua: Develando sus orígenes híbridos en la cuenca del Río Bueno, Chile. Revista de Geografía Norte Grande, 73, 9-27. https:// doi.org/10.4067/S0718-34022019000200009

Organización Mundial de la Salud (oms). (2003). oms | La cantidad de agua domiciliaria, el nivel del servicio y la salud. World Health Organization (wHO). https://www.who.int/ water_sanitation_health/diseases/wsh0302/es/

Panez-Pinto, A., Faúndez-Vergara, R. \& Mansilla-Quiñones, C. (2017). Politización de la crisis hídrica en Chile: Análisis del conflicto por el agua en la provincia de Petorca. Agua y Territorio / Water and Landscape, 10, 131-148. https://doi.org/10.17561/at.10.3614

Perramond, E. P. (2016). Adjudicating hydrosocial territory in New Mexico. Water International, 41(1), 173-188. https://doi.org/10.1080/02508060.2016.1108442

Prieto, M. (2015). La ecología (a)política del modelo de aguas chileno. En B. Bustos, M. Prieto \& J. Barton, Ecología politica en Chile: Naturaleza, propiedad, conocimiento y poder (pp. 143-166). Editorial Universitaria, Chile.

Seemann, M. (2016). Inclusive recognition politics and the struggle over hydrosocial territories in two Bolivian highland communities. Water International, 41(1), 157-172. https:// doi.org/10.1080/02508060.2016.1108384

Subsecretaría del Interior, Chile. (2016). Oficio Ordinario $n^{\circ}$ 18.087. Ministerio del Interior, Chile. https://ciperchile.cl/pdfs/2017/marzo/OFICIO_INTERIOR_\%2018.087.pdf 
Swyngedouw, E. \& Boelens, R. (2018). “... And not a single injustice remains”: Hydroterritorial colonization and techno-political transformations in Spain. En J. Vos, R. Boelens \& T. Perreault (Eds.), Water Justice (pp. 115-133). Cambridge University Press. https://doi.org/10.1017/9781316831847.008

Urquiza, A. \& Billi, M. (2020). Water markets and social-ecological resilience to water stress in the context of climate change: An analysis of the Limarí Basin, Chile. Environment, Development and Sustainability, 22(3), 1929-1951. https://doi.org/10.1007/s10668018-0271-3

Wester, P. (2008). Shedding the waters: Institutional change and water control in the LermaChapala Basin, Mexico. Wageningen University. 